\title{
Solvent-free preparation of primary carbamates using silica sulfuric acid as an efficient reagent
}

\author{
Ali Reza Modarresi-Alam,* Mahmoud Nasrollahzadeh, and Ferydoon Khamooshi \\ Department of Chemistry, University of Sistan \& Baluchestan, Zahedan, Iran \\ E-mail:modaresi@hamoon.usb.ac.ir
}

\begin{abstract}
A simple and efficient method for the conversion of structurally diverse compounds containing a hydroxyl group to primary carbamates is described by grindstone chemistry. The transformation was done at room temperature with high yield and purity, and without any epimerization in the absence of solvent. Silica sulfuric acid was used as a solid acid and as a mild, convenient and effective reagent for this transformation.
\end{abstract}

Keywords: Grindstone chemistry, solvent-free conditions, N-unsubstituted carbamates, silica sulfuric acid

\section{Introduction}

Carbamates (urethanes) are compounds of growing interest because of their applications in the agrochemicals industry $^{1-5}$ as herbicides, fungicides and pesticides, in the pharmaceuticals industry $^{1,2,6}$ as drug intermediates and in the polymer industry, ${ }^{1,2,7}$ in the synthesis of polyurethane and peptides. In addition, among the various amine-protecting groups, carbamates are commonly used due to their chemical stability towards acids, bases and hydrogenation. ${ }^{8}$ These materials are most commonly prepared from amines and alcohols by carbonylation using phosgene in organic solvents which are also toxic and flammable. ${ }^{1-3}$ These procedures seem to be efficient, pose environmental and operational concerns since highly harmful and corrosive reagents are used. Efforts have been continuously made for the replacement of the phosgene with carbon dioxide and organic carbonates. ${ }^{9}$ However these methods cannot produce $N$-unsubstituted (primary) carbamates. Synthesis of $N$-unsubstituted carbamates $\mathbf{1}$ from alcohols has been also accomplished by several-pot reaction methods such as; trichloroacetyl isocyanate, ${ }^{10,11}$ chloroformates (starting from toxic phosgene), ${ }^{12}$ chlorosulfonyl isocyanate $^{13}$ and cyanogen chloride. $^{14}$ 
Loev and coworkers reported the synthesis of $N$-unsubstituted carbamates from alcohols by treatment with sodium cyanate and trifluoroacetic acid in certain organic solvents such as benzene, methylene chloride and carbon tetrachloride without any spectral data such as IR and NMR. ${ }^{15}$ These solvents are toxic and are not eco-friendly. In addition, trifluoroacetic acid is very expensive. From the standpoint of 'green chemistry', significant efforts have been made to find an alternative to organic solvents. A very attractive substitute for these solvents is a solvent-free reaction (industrially important due to reduced pollution, low cost, and simplicity in process and handling). ${ }^{16-21}$

Grindstone Chemistry is a branch of green chemistry for solvent-free chemical reactions which can be probably conducted in high yield by just grinding solid/solid, solid/liquid, or even liquid/liquid together. ${ }^{22}$ Required activation energy is provided from friction of the reactive molecules in solvent-free conditions.

In attempts to synthesize primary carbamates from phenols and alcohols under solvent-free conditions, we have recently reported a method for the conversion of compounds containing hydroxyl group to primary carbamates at room temperature in the absence of solvent using trichloroacetic acid as well as spectra data such as IR, NMR and their dynamic NMR. ${ }^{23,24}$ Since this acid is relatively toxic and corrosive, we were interested in developing methods for the synthesis of carbamates utilizing solid acids such as $\mathrm{HClO}_{4}-\mathrm{SiO}_{2},{ }^{25}$ as they are industrially important due to their potential at replacing conventional acid/base catalysts. ${ }^{19-21,26-29}$

The use of solid acids such as silica sulfuric acid (SSA) for synthesizing organic intermediates and fine chemicals is gaining awareness increasingly, also, making a field of intense research activity. ${ }^{30}$ Furthermore, in the past decade the development of new technologies have been expedited to strive to eliminate the need for chromatographic separation of mixtures, especially impurities and this itself has led to the development of new technologies in synthetic organic chemistry. ${ }^{31}$

In this paper, a simple and efficient solvent-free methodology was performed to prepare primary carbamates $\mathbf{1}$ in high yield and purity from compounds $\mathbf{2}$, sodium cyanate and silica sulfuric acid (Scheme 1 and 2). However, to the best of our knowledge there has been no report on the use of silica sulfuric acid for primary carbamates synthesis.

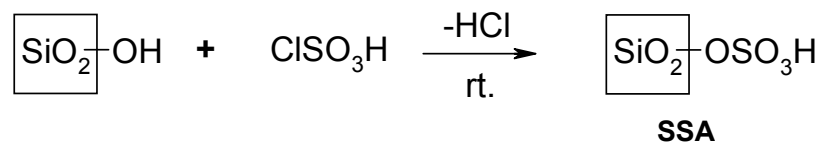

\section{Scheme 1}




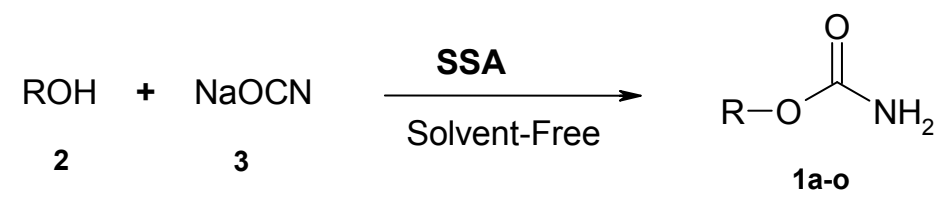

\section{Scheme 2}

\section{Results and Discussion}

As mentioned in the literature, ${ }^{30}$ silica sulfuric acid (SSA) was obtained from silica gel and chlorosulfonic acid (Scheme 1).

Primary carbamates 1a-o were prepared in high yields and in high purity from reaction of either alcohol or phenol $\mathbf{2}$ with sodium cyanate $\mathbf{3}$ in the presence of SSA at room temperature or 55-65 ${ }^{\circ} \mathrm{C}$ for the appropriate time, as showed in Table 1 and Scheme 2.

As indicated in Table 1 some substrates with various structures were used to synthesize primary carbamates 1a-o purely and cleanly through this easy procedure. Primary, secondary, tertiary, allylic, benzylic alcohols and phenols showed a smooth conversion to the corresponding carbamates. It is high important to mention that (-)-menthol 2a the reaction produced the corresponding (-)-menthyl carbamate 1a while no epimerization took place. The crude product, in most cases, was completely pure and did not require to be purified or worked up anymore. Since the activation energy is low for alcohols 1a-i then their carbamates formation is very easy. The activation energy is provided from friction of available molecules in solid phase (alcohols $\mathbf{1 a - i}$, sodium cyanate and silica sulfuric acid). However activation energy of phenols $\mathbf{1} \mathbf{j}-\mathbf{0}$ is higher than alcohols 1a-i, so the yield and purity of aromatic compounds $\mathbf{1 j - 0}$ (entries 10-15) was modified by heating at $55-65^{\circ} \mathrm{C}$ for $1 \mathrm{~h}$. The low nucleophilicity of the phenol oxygen is a reason of this difference. Phenols which carried electron-withdrawing substituents (CN, COOR and $\mathrm{CHO}$ ) did not react successfully in our experimental conditions. These substituents very probably reduce the nucleophilicity of the phenol oxygen, so they fail to attack intermediate $\mathbf{5}$ and/or 7 (Scheme 3). This might have caused low yield (60\%) of compound 11 (entry 12). Moreover, we found that the reaction conditions could tolerate such moieties as $O$ - $i$-propyl (1h, entry 8) which often undergoes cleavage in strongly acidic media. In addition, it is remarkable to note that reaction of $\alpha$ - and $\beta$-naphthol (entries 14 and 15) proceeded effectively in the present experimental conditions, while it did not work completely in trichloroacetic acid (the latest reported procedure ${ }^{23,24}$ ). Indeed, the amount of remained starting material after long times and/or at high temperatures was really considerable. Moreover, regarding removal of trichloroacetic acid and reaction time, the present procedure was more effective than what recently reported, ${ }^{23,24}$ because the time was shortened from $12 \mathrm{hr}$ to $1 \mathrm{hr}$ (Table 1 ). 
By comparing the IR and physical properties of the products with those of authentic samples, ${ }^{23,25,32-36}$ carbamates 1a-o were easily identified. Also; they were characterized by ${ }^{1} \mathrm{H}-$ NMR $(500 \mathrm{MHz})$ and ${ }^{13} \mathrm{C}-\mathrm{NMR}(125 \mathrm{MHz})$. The signals of carbonyl carbon of aliphatic or aromatic carbamate is displayed in ${ }^{13} \mathrm{C}$-NMR spectra in the range of $\delta 146-157 \mathrm{ppm}$.

The present feasible reaction mechanism resembles the recently reported one ${ }^{23,25}$ which is illustrated in Scheme 3. The reaction of sodium cyanate 3 with an acid (SSA) to produce isocyanic acid $\mathbf{5}$ could be the first step. ${ }^{23,37,38} \mathrm{Next}$, for the generation of the intermediate $\mathbf{7}$, the proton of SSA is added to isocyanic acid $\mathbf{5}$ that the proton is perfectly added to nitrogen rather than oxygen. ${ }^{39}$ Finally, carbamate $\mathbf{1}$ is likely to be formed when either alcohol or phenol $\mathbf{2}$ attack to the carbon of the intermediate 7 (Scheme 3).

In conclusion, this simple solvent free method affords various primary carbamates at room temperature in short reaction times, with high yields and purity, without involvement of toxic solvents, expensive starting materials, formation of any undesirable side products and epimerization. Also, we have revealed that silica sulfuric acid is a highly effective reagent for synthesizing primary carbamates. Furthermore, this method does not require purification or separation techniques (column chromatography). Further studies are in progress.

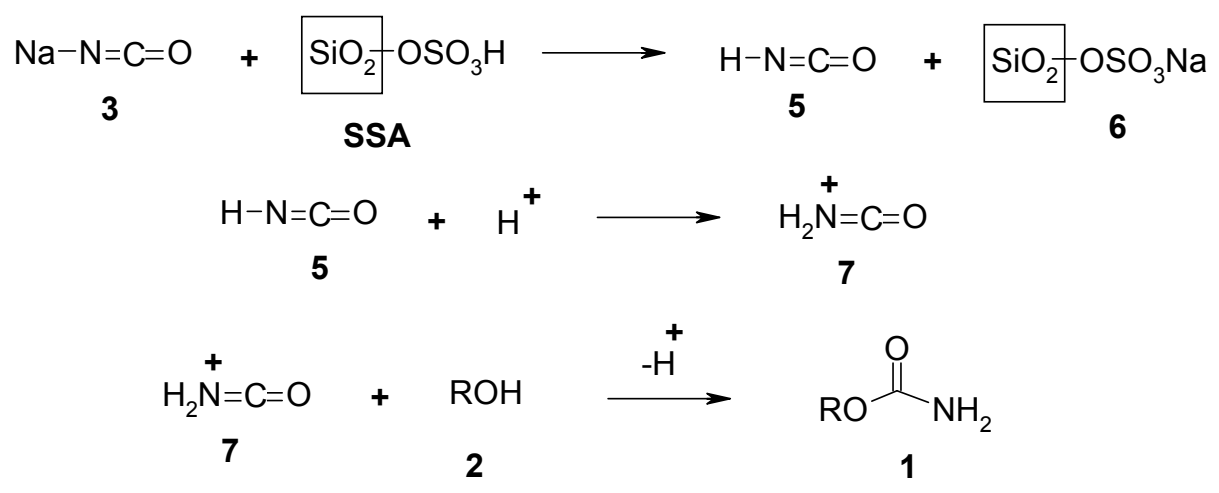

\section{Scheme 3}


Table 1. Preparation of primary carbamates 1a-0 $\mathbf{a}^{\mathrm{a}}$

\begin{tabular}{crlcc}
\hline Entry & Compound & \multicolumn{1}{c}{$\mathrm{R}$} & Time (min) & Yield (\%) \\
\hline 1 & $\mathbf{1 a}$ & $(-)-\mathrm{Menthyl}$ & 50 & 81 \\
2 & $\mathbf{1 b}$ & $\mathrm{CH}_{3} \mathrm{CH}_{2}$ & 50 & 67 \\
3 & $\mathbf{1 c}$ & $\mathrm{CH}_{3} \mathrm{CH}_{2} \mathrm{CH}_{2}$ & 50 & 82 \\
4 & $\mathbf{1 d}$ & $\mathrm{CH}_{3} \mathrm{CH}_{2} \mathrm{CH}_{2} \mathrm{CH}_{2}$ & 50 & 73 \\
5 & $\mathbf{1 e}$ & $\mathrm{C}_{6} \mathrm{H}_{11}$ & 50 & 76 \\
6 & $\mathbf{1 f}$ & $\left(\mathrm{CH}_{3}\right)_{3} \mathrm{C}$ & 50 & 70 \\
7 & $\mathbf{1 g}$ & $\mathrm{PhCH}_{2}$ & 50 & 72 \\
8 & $\mathbf{1 h}$ & $\left(\mathrm{CH}_{3}\right)_{2} \mathrm{CHOCH}_{2} \mathrm{CH}_{2}$ & 50 & 80 \\
9 & $\mathbf{1 i}$ & $\mathrm{CH}_{2}=\mathrm{CHCH}_{2}$ & 50 & 75 \\
10 & $\mathbf{1 j}$ & $\mathrm{C}_{6} \mathrm{H}_{5}{ }^{\mathrm{b}}$ & 60 & 82 \\
11 & $\mathbf{1 k}$ & $4-\mathrm{CH}_{3} \mathrm{C}_{6} \mathrm{H}_{4}{ }^{\mathrm{b}}$ & 60 & 81 \\
12 & $\mathbf{1 1}$ & $4-\mathrm{BrC}_{6} \mathrm{H}_{4}{ }^{\mathrm{b}}$ & 60 & 60 \\
13 & $\mathbf{1 m}$ & $\left.2-\mathrm{C}_{(\mathrm{CH}}\right)_{3}-4-\mathrm{CH}_{3} \mathrm{C}_{6} \mathrm{H}_{4}{ }^{\mathrm{b}}$ & 60 & 78 \\
14 & $\mathbf{1 n}$ & $\alpha-\mathrm{naphthyl}^{\mathrm{b}}$ & 60 & 77 \\
15 & $\mathbf{1 0}$ & $\beta-\mathrm{naphthyl}^{\mathrm{b}}$ & 60 & 85 \\
\hline
\end{tabular}

${ }^{\mathrm{a}}$ See ref. 23, 25 and 32-36 for carbamates 1a-o. ${ }^{\mathrm{b}}$ By heating at $55-65{ }^{\circ} \mathrm{C}$ for $1 \mathrm{~h}$.

\section{Experimental Section}

General Procedures. ${ }^{1} \mathrm{H}-\mathrm{NMR}$ and ${ }^{13} \mathrm{C}-\mathrm{NMR}$ spectra were recorded by BRUKER AVANCE DRX500 (500 MHz). The IR spectra were obtained on a SHIMADZU-470. Melting points were recorded by Electro thermal 9100 and were uncorrected. Thin layer chromatography (TLC) was carried out using plastic sheets precoated with silica gel $60 \mathrm{~F}$. All starting materials such as alcohols, phenols, NaOCN and solvents were purchased from Fluka, Merck and Aldrich chemical companies and were purified with the proper purification techniques before use. ${ }^{40,41}$ The products 1 were identified through comparison of their spectral data, IR, ${ }^{1} \mathrm{H}-\mathrm{NMR}$, ${ }^{13} \mathrm{C}$-NMR, TLC and physical properties with those of authentic samples. ${ }^{23,25,32-36}$ Silica sulfuric acid (SSA) was prepared from silica gel and chlorosulfonic acid according to literature. ${ }^{30}$

\section{General preparative procedure}

In a typical procedure, to a mixture of sodium cyanate $(2 \mathrm{mmol})$ and SSA $(0.77 \mathrm{~g}, 2 \mathrm{mmol})$, alcohol or phenol $(1.0 \mathrm{mmol})$ was added and the mixture was pulverized in a mortar (or the mixture can be also stirred by a magnet in a test tube) at room temperature or $55-65{ }^{\circ} \mathrm{C}$ for appropriate time (Table 1). The reaction was monitored in TLC. After completion of reaction, $\mathrm{CHCl}_{3}$ was added and the mixture was filtered for separating of reagent. The solvent $\left(\mathrm{CHCl}_{3}\right)$ evaporated to give product. Pure products were obtained in high yields, as summarized in Table 
1. In cases of $\alpha$ - and $\beta$-naphthol (entries 14 and 15) after removing $\mathrm{CHCl}_{3}$, petroleum ether and then ethyl acetate were added. The obtained solid was pure $\alpha$ - or $\beta$-naphthyl carbamate $1 \mathbf{n}$ and 10.

Naphthalen-1-yl carbamate (1n). White crystals 1n (77\%), mp $=178-180{ }^{\circ} \mathrm{C}^{32} \mathrm{IR}\left(\mathrm{KBr}, \mathrm{cm}^{-1}\right)$ : 3430 (m), 3343 (vw), 3275 (w), 3200 (w), 3055 (vw), 2920 (vw), 1698 (vs), 1603 (s), 1360 (vs), $1254(\mathrm{~s}), 1222(\mathrm{~s}), 1150(\mathrm{~m}), 1082(\mathrm{~s}), 1041(\mathrm{~m}), 1010(\mathrm{~m}), 958(\mathrm{~m}), 801(\mathrm{~s}), 773(\mathrm{vs}), 582(\mathrm{~m})$, 553 (w). ${ }^{1} \mathrm{H}-\mathrm{NMR}\left(500 \mathrm{MHz}, \mathrm{CDCl}_{3}\right), \delta \mathrm{ppm} ; 6.10$ (br, d, 2H), $7.20(\mathrm{~d}, \mathrm{~J}=7.5 \mathrm{~Hz}, 1 \mathrm{H}), 7.35-$ $7.45(\mathrm{~m}, 3 \mathrm{H}), 7.63(\mathrm{~d}, \mathrm{~J}=8.2 \mathrm{~Hz}, 1 \mathrm{H}), 7.78(\mathrm{dd}, \mathrm{J}=9.3 \mathrm{~Hz}, \mathrm{~J}=2.1 \mathrm{~Hz}, 1 \mathrm{H}), 7.92(\mathrm{dd}, \mathrm{J}=8.8$ $\mathrm{Hz}, \mathrm{J}=2.1 \mathrm{~Hz}, 1 \mathrm{H}) .{ }^{13} \mathrm{C}-\mathrm{NMR}\left(125 \mathrm{MHz}, \mathrm{CDCl}_{3}\right), \delta \mathrm{ppm} ; 117.7,120.8,124.8,124.9,125.6$, 125.7, 126.9, 127.3, 134.0, 146.2, 154.7. Analysis Calcd. For $\mathrm{C}_{11} \mathrm{H}_{9} \mathrm{NO}_{2}$ : C, 70.59; H, 4.81; N, 7.49; Found; C, 70.80; H, 4.71; N, 7.60\%.

Naphthalen-2-yl carbamate (10). White crystals $10(85 \%), \mathrm{mp}=157-158{ }^{\circ} \mathrm{C} .{ }^{33} \mathrm{IR}\left(\mathrm{KBr}, \mathrm{cm}^{-1}\right)$ : 3405 (m), 3038 (w), 3270 (w), 3197 (vw), 3055 (vw), 1697 (vs), 1610 (w), 1506 (w), 1388 (s), 1355 (s), 1239 (s), $1206(\mathrm{~s}), 1155(\mathrm{~m}), 987$ (s), 895 (m), 858 (m), 821 (m), $775(\mathrm{~m}), 758(\mathrm{w}), 734$ (m), $543(\mathrm{w}), 474(\mathrm{~m}) .{ }^{1} \mathrm{H}-\mathrm{NMR}\left(500 \mathrm{MHz}, \mathrm{CDCl}_{3}\right.$ ), $\delta \mathrm{ppm} ; 6.25$ (br, s, 2H), 7.20 (dd, J = 8.7 $\mathrm{Hz}, \mathrm{J}=2.1 \mathrm{~Hz}, 1 \mathrm{H}), 7.34-7.41(\mathrm{~m}, 2 \mathrm{H}), 7.49(\mathrm{~d}, \mathrm{~J}=2.1 \mathrm{~Hz}, 1 \mathrm{H}), 7.71(\mathrm{~d}, \mathrm{~J}=8.0 \mathrm{~Hz}, 1 \mathrm{H}), 7.75$ $(\mathrm{d}, \mathrm{J}=8.7 \mathrm{~Hz}, 2 \mathrm{H}) .{ }^{13} \mathrm{C}-\mathrm{NMR}\left(125 \mathrm{MHz}, \mathrm{CDCl}_{3}\right), \delta \mathrm{ppm} ; 117.9,121.2,124.8,125.8,126.9$, 127.1, 128.5, 130.5, 133.1, 148.3, 154.9, Analysis Calcd. For $\mathrm{C}_{11} \mathrm{H}_{9} \mathrm{NO}_{2}$ : C, 70.59; H, 4.81; N, 7.49; Found; C, 71.20; H, 4.65; N, 7.54\%.

Menthyl carbamate 1a, $\mathrm{mp}=166-168^{\circ} \mathrm{C}$ (lit. $\left.{ }^{25,34} 156-157^{\circ} \mathrm{C}\right)$. Ethyl carbamate 1b, $\mathrm{mp}=46-$ $48{ }^{\circ} \mathrm{C}$ (lit. ${ }^{25,35} 48-50{ }^{\circ} \mathrm{C}$ ). 1-Propyl carbamate 1c, $\mathrm{mp}=58-59{ }^{\circ} \mathrm{C}$ (lit. ${ }^{25,35} 60{ }^{\circ} \mathrm{C}$ ). 1-Buthyl carbamate 1e , $\mathrm{mp}=53-55^{\circ} \mathrm{C}\left(\right.$ lit. ${ }^{25,35} 54^{\circ} \mathrm{C}$ ). Cyclohexyl carbamate 1e, $\mathrm{mp}=108-110^{\circ} \mathrm{C}$ (lit. ${ }^{23,25} 108-110{ }^{\circ} \mathrm{C}$ ). tert-Buthyl carbamate 1f, $\mathrm{mp}=106-108^{\circ} \mathrm{C}$ (lit. ${ }^{15,25} 107-108{ }^{\circ} \mathrm{C}$ ). Benzyl carbamate 1g, $\mathrm{mp}=87-89{ }^{\circ} \mathrm{C}$ (lit. ${ }^{25,35} 91{ }^{\circ} \mathrm{C}$ ). Ethylene glycol monoisopropyl ether carbamate $1 \mathbf{h}, \mathrm{mp}=57-59{ }^{\circ} \mathrm{C}\left(\right.$ lit. ${ }^{25,36} 53{ }^{\circ} \mathrm{C}$ ). Allyl carbamate 1i, $\mathrm{mp}=19-21{ }^{\circ} \mathrm{C}$ (lit. ${ }^{23,25} 19$ $21{ }^{\circ} \mathrm{C}$ ). Phenyl carbamate $\mathbf{1 j}$, $\mathrm{mp}=141-143{ }^{\circ} \mathrm{C}$ (lit. ${ }^{16,25} 145-148{ }^{\circ} \mathrm{C}$ ). 4-Methylphenyl carbamate $1 \mathbf{k}, \mathrm{mp}=134-136{ }^{\circ} \mathrm{C}$ (lit. ${ }^{23,25} 134-136{ }^{\circ} \mathrm{C}$ ). 4-Bromophenyl carbamate $1 \mathbf{1}, \mathrm{mp}=$ 139-142 ${ }^{\circ} \mathrm{C}$ (lit. ${ }^{23,25} 139-142{ }^{\circ} \mathrm{C}$ ). 2-tert-Butyl-4-Methylphenyl carbamate 1m, mp = 143-144 ${ }^{\circ} \mathrm{C}$ (lit. ${ }^{23,25} 143-144{ }^{\circ} \mathrm{C}$ ). Naphthalen-1-yl carbamate 1n, $\mathrm{mp}=178-180{ }^{\circ} \mathrm{C}$ (lit. ${ }^{25,32}$ 175-177 $\left.{ }^{\circ} \mathrm{C}\right)$. Naphthalen-2-yl carbamate 1o, $\mathrm{mp}=157-158^{\circ} \mathrm{C}\left(\right.$ lit. $\left.{ }^{25,33} 156-157^{\circ} \mathrm{C}\right)$.

\section{Acknowledgements}

The Sistan \& Baluchestan University Graduate Council supported this research. 


\section{References}

1. Dibenedetto, A.; Aresta, M.; Fragale, C.; Narracci, M. Green Chemistry 2002, 4, 439 and references therein.

2. Gupte, S. P.; Shivarkar, A. B.; Chaudhari, R. V. J. Chem. Soc., Chem. Commun. 2001, 2620.

3. Mindl, J.; Hrabík, O.; Stěrba, V.; Kaválek, J. Collect. Czech. Chem. Commun. 2000, 65, 1262.

4. Motolcsy, G. ; Nadasy, M.; Andriska, V. Pesticide Chemistry; Academiai Kiado: Budapest, 1988; pp 90.

5. Thompson, A. Pesticide Outlook 2002, 13, 84.

6. Martin, L. L.; Davis, L.; Klein, J. T.; Nemoto, P.; Olsen, G. E.; Bores, G. M.; Camacho, F.; Petko, W. W.; Rush, D. K.; Selk, D.; Smith, C. P.; Vargas, H. M.; Wilson, J. T.; Effland, R. C.; Fink, D. M. Bioorg. Med. Chem. Lett. 1997, 7, 157.

7. Feldman, D.; Barbalata, A. Synthetic Polymers, Technology, Properties, Applications; Chapman and Hall: London, 1996; pp 273.

8. Greene, W. T.; Wuts, P. G. M. Protective Groups in Organic Synthesis, $2^{\text {nd }}$ Edn.; Wiley: New York, 1991; pp 327 and 403.

9. Yoshida, M.; Hara, N.; Okuyama, S. J. Chem. Soc., Chem. Commun. 2000, 151 and references therein.

10. Ichikawa, Y.; Osada, M.; Ohtani, I. I.; Isobe, M. J. Chem. Soc., Perkin Trans. 1 1997, 1449.

11. Kocovsky, P. Tetrahedron Let. 1986, 27, 5521.

12. Raucher, S.; Jones, D. S. Synth. Commun. 1985, 15, 1025.

13. Graf, R. Ber. 1963, 96, 56.

14. Fuks, R.; Hartemink, M. A. Bull. Chim. Belg. 1973, 82, 23.

15. Loev, B; Kormendy, M. F. J. Org. Chem. 1963, 28, 3421.

16. Tanaka, K. Solvent-Free Organic Synthesis; Wiley-VCH: Morlenbach, 2003.

17. Nagendrappa, G. Resonance 2002, 7, 64.

18. Varma, R. S. Green Chemistry 1999, 1, 43.

19. Varma, R. S. Tetrahedron 2002, 58, 1235.

20. Tanaka, K.; Toda, F. Chem. Rev. 2000, 100, 1025.

21. (a) Clark, J. H.; Macquarrie, D. J. Chem. Soc. Rev. 1996, 25, 303. (b) Clark, J. H.; Cullen, S. R.; Barlow, S. J.; Bastock, T. W. J. Chem. Soc., Perkin Trans. 2 1994, 1117. (c) Clark, J. H.; Macquarrie, D. J. Chem. Commun. 1998, 853.

22. Bose, A. K.; Pednekar, S.; Ganguly, S. N.; Chakraborty, G.; Manhas, M. S. Tetrahedron Lett. 2004, 45, 8351.

23. Modarresi-Alam, A. R.; Rostamizadeh, M.; Najafi, P. Turk. J. Chem. 2006, 30, 269.

24. Modarresi-Alam, A. R.; Najafi, P.; Rostamizadeh, M.; Keykha, H.; Bijanzadeh, H.-R.; Kleinpeter, E. J. Org. Chem. 2007, 72, 2208.

25. Modarresi-Alam, A. R.; Khamooshi, F.; Nasrollahzadeh, M.; Amiraziz, H. A. Tetrahedron 2007, 63, 8723. 
26. Melero, J. A.; Grieken, R. V.; Morales, G. Chem. Rev. 2006, 106, 3790.

27. Yadav, G. D. Catalysis Surveys from Asia 2005, 9, 117.

28. Okuhara, T. Chem. Rev. 2002, 102, 3641.

29. Gorte, R. J. Catalysis Letters 1999, 62, 1.

30. (a) Thirunarayanan, G.; Vanangamudi, G. ARKIVOC 2006, (xii), 58. (b) Pore, D. M.; Desai, U. V.; Thopate, T. S.; Wadgaonkar, P. P. ARKIVOC 2006, (xii), 75. (c) Zolfigol, M. A. Tetrahedron 2001, 57, 9509. (d) Salehi, P.; Dabiri, M.; Zolfigol, M. A.; Bodaghi Fard, M. A. J. Braz. Chem. Soc. 2004, 15, 773. (e) Mirjalili, B. F.; Zolfigol, M. A.; Bamoniri, A.; Zarei, A. J. Chines. Chem. Soc. 2004, 51, 509. (f) Zolfigol, M. A.; Mirjalili, B. F., Bamoniri, A.; Karimi Zarchi, M. A.; Zarei, A.; Khazdooz, L.; Noei, J. Bull. Korean Chem. Soc. 2004, 25, 1414. (g) Salehi, P.; Dabiri, M.; Zolfigol, M. A.; Bodaghi Fard, M. A. Heterocycle 2003, 60, 2435. (h) Mirjalili, B. F.; Zolfigol, M. A.; Bamoniri, A.; Zaghaghi, Z. J. Chem. Research (S) 2003, 273. (i) Mirjalili, B. F.; Zolfigol, M. A.; Bamoniri, A.; Zaghaghi, Z.; Hazar, A. Acta Chem. Slov. 2003, 50, 563. (j) Salehi, P.; Dabiri, M.; Zolfigol, M. A.; Bodaghi Fard, M. A. Tetrahedron Lett. 2003, 44, 2889. (k) Zolfigol, M. A.; Chehardoli, G.; Mallakpour, S. E. Synth. Commun. 2003, 33, 833. (1) Zolfigol, M. A., Shirin, F.; Ghorbani Choghamarani, A.; Mohammadpoor-Baltork, I. Green Chem. 2002, 4, 562. (m) Zolfigol, M. A., Bamoniri, A. Synlett. 2002, 1621. Moore, J. D.; Herpel, R. H.; Lichtsinn, J. R.; Flynn, D. L.; Hanson, P. R. Org. Lett. 2003, 5, 105 and references therein.

31. Fahmy, M. A. H.; Fukuto, T. R. J. Agr. Food Chem. 1972, 20, 168.

32. Barenes, J. H.; Chapman, M. V. A.; McCrea, P. A.; Marshall, P. G.; Walsh, P. A. J. Pharm. Pharmacol. 1961, 13, 39.

33. Lowenthal, M. S.; Khanna, R. K.; Moore, M. H. Spectrochimia Acta 2002, Part 58 A, 73.

34. Oshikawa, T.; Yamashita, M. Bull. Chem. Soc. Jpn. 1989, 62, 3177.

35. Weast, R. C.; Lide, D. R. Handbook of Chemistry and Physics, 70th Edn., CRC Press: Boca Raton, Florida, 1989-1990, C-194.

36. Ashburn, H. G.; Collett, A. R.; Lazzell, C. L. J. Am. Chem. Soc. 1938, 60, 2933.

37. Lowenthal, M. S.; Khanna, R. K.; Moore, M. H. Spectrochimia Acta 2002, Part 58 A, 73.

38. Fischer, G.; Geith, J.; Klapotke, T. M.; Krumm, B. Naturforsch. 2002, 57b, 19.

39. (a) Kabir-ud-Din; Owen, R. C.; Fox, M. A. J. Phys. Chem. 1981, 85, 1679. (b) Nguyen, M. T.; Sengupta, D.; Vereecken, L.; Peeters, J.; Vanquickenborne, L. G. J. Phys. Chem. 1996, $100,1615$.

40. Casey, M.; Leonard, J.; Lygo, B.; Proctor, G. Advanced Practical Organic Chemistry, Chapman \& Hall, Int.: New York, 1990.

41. Armarego, W. L. F.; Perrin, D. D. Purification of Laboratory Chemicals, ButterworthHeinmam: Oxford, 1996. 